\title{
EL BOOKTRAILER COMO ESTRATEGIA DIDÁCTICA PARA EL DESARROLLO DE COMPETENCIAS COMUNICATIVAS, NARRATIVAS Y DIGITALES
}

\section{THE BOOKTRAILER AS A DIDACTIC STRATEGY FOR THE DEVELOPMENT OF COMMUNICATIVE, NARRATIVE AND DIGITAL COMPETENCES}

\author{
Lina Yormary Martínez Diaz \\ Ariel Adolfo Rodríguez Hernández ${ }^{2}$ \\ Universidad Pedagógica y Tecnológica de \\ Colombia, Tunja, Colombia
}

\section{RESUMEN}

En este trabajo se reportan los resultados de un estudio el cual tuvo por objeto validar la $\overline{1}$ Magister en TIC Aplicadas a las Ciencias de la Educación, Licenciada en tecnología, Docente, Universidad Pedagógica y Tecnológica de Colombia. linayormary.martinez@uptc.edu.co .ORCID ID: https://orcid.org/0000-00016079-412X

2 Magíster en Gestión Pedagógica, Ingeniero en Sistemas con Énfasis en Software Docente investigador, Universidad Pedagógica y Tecnológica de Colombia. ariel. rodriguez@uptc.edu.co. ORCID ID: https://orcid.org/00000003-1906-7734

efectividad del booktrailer como una estrategia didáctica para el desarrollo de competencias comunicativas, narrativas y digitales en educación primaria. El estudio se desarrolló bajo un enfoque mixto de tipo cuasiexperimental y la población objeto de estudio correspondió a 74 estudiantes de grado cuarto de primaria pertenecientes a una institución educativa colombiana. Los resultados evidencian que 72 estudiantes lograron adquirir competencias 
digitales y narrativas y tan solo a 3 estudiantes se les dificultó desarrollar la competencia comunicativa. Se concluye que el booktrailer como estrategia didáctica, tiene un alto nivel de aceptabilidad para su implementación en el área de literatura y en el área de tecnología e informática.

PALABRAS CLAVE: competencias comunicativas, competencias narrativas, competencias digitales, booktrailer, educación infantil.

\section{ABSTRACT}

This work reports the results of a study which aimed to validate the effectiveness of the booktrailer as a didactic strategy for the development of communicative, narrative and digital skills in primary education. The study was carried out using a mixed quasi-experimental approach and the population under study corresponded to 74 fourth grade students from a Colombian educational institution. The results show that 72 students managed to acquire digital and narrative skills and only 3 students found it difficult to develop communication skills. It is concluded that the booktrailer as a didactic strategy has a high level of acceptability for its implementation in the literature area and in the technology and computer science area.

KEYWORDS: communication skills, narrative skills, digital skills, booktrailer, early childhood education.

\section{INTRODUCCIÓN}

En la actualidad, el sector educativo presenta grandes retos que exigen a docentes $y$ estudiantes el desarrollo y adaptación de nuevas estrategias pedagógicas para la enseñanzaaprendizaje de conceptos en diferentes áreas del conocimiento (Barrera-Mesa, FernándezMorales \& Duarte, 2017; López-Gaitán, Moran-Borbor, \& Niño-Vega, 2018; JiménezEspinosa \& Sánchez-Bareño, 2019). A ello, la implementación de las Tecnologías de la Información y la Comunicación, TIC, han venido tomando auge en la educación, gracias a la fácil adquisición de dispositivos tecnológicos, a las múltiples herramientas informáticas desarrolladas y al diverso contenido digital que se dispone en la red (Martínez-López \& Gualdrón-Pinto, 2018; Bernate et al., 2020).

El incorporar las TIC en la educación ha traído consigo grandes beneficios a docentes, debido a que facilita la enseñanza de conceptos complejos (Ruiz-Macías \& Duarte, 2018; Niño-Vega et al., 2017), así como les permite emplear recursos digitales idóneos según las necesidades de aprendizaje de los estudiantes (Niño-Vega, Fernández-Morales \& Duarte, 2019; Jiménez-Espinosa, 2019). Del mismo modo, las TIC favorecen a los estudiantes en el proceso de aprendizaje, ya que permiten adquirir conocimientos de manera más interactiva; les permiten retroalimentar saberes preconcebidos por medio de la experimentación y la gamificación, aumentando su interés y motivación por aprender (Vargas-Vargas, NiñoVega \& Fernández-Morales, 2020; FonsecaBarrera et al, 2020).

Por otra parte, el desarrollo de competencias comunicativas narrativas y digitales ha de ser un lineamiento básico en educación primaria, ya que permite al estudiante mejorar su capacidad de comprensión, análisis y comunicación ya sea de forma oral o escrita en escenarios reales o virtuales (Niño-Vega \& Fernández- Morales, 2019; Naranjo-Gaviria \& Herreño-Téllez, 2020). A lo expuesto, en la educación literaria y lingüística se ha comprobado que las narrativas digitales tienen un alto impacto en el desarrollo de estas competencias, debido a que permiten representar escenas a través de múltiples formatos como: imágenes, videos, audios y textos, el cual funciona como herramienta digital para potencializar el aprendizaje lecto- 
escritor (Salcedo-Salcedo \& Fernández, 2018; Hernández-Gil \& Jaramillo-Gaitán, 2020).

De acuerdo con Rovira (2017), los Booktrailer se conciben como propuestas de promoción multimedia de las obras literarias que pueden utilizarse como estrategia publicitaria de una editorial o como propuesta didáctica a trabajar dentro del aula y la motivación por la lectura (Avella-lbáñez, Sandoval-Valero \& MontañezTorres,2017). Dentro del aula estas estrategias se convierten en una atractiva herramienta metodológica para la didáctica de la lectura y la literatura contemporánea, puesto que permite trabajar adquisición de la competencias literaria, comunicativa, digital y mediática desde la comprensión y producción de un texto (Ibarra \& Ballester, 2016).

Con lo anterior, en este artículo se presentan los resultados obtenidos luego de aplicar una estrategia didáctica basado en booktrailer y apoyadas con herramientas TIC, las cuales buscan desarrollar competencias comunicativas, narrativas y digitales de los estudiantes de cuarto grado de primaria. A continuación, se enuncia el marco teórico relacionado con las competencias a desarrollar y la metodología empleada en la ejecución de esta investigación. Posteriormente se describe los resultados obtenidos de este estudio. Finalmente, se detallan las conclusiones obtenidas a partir de la implementación de la estrategia didáctica propuesta con la población objeto de estudio.

\section{MARCO TEÓRICO Y METODOLOGÍA}

\subsection{Estrategia didáctica mediadas por TIC}

las estrategias didácticas resultan ser un conjunto de acciones que establece el docente para mejorar el proceso de enseñanza-aprendizaje y atender a problemas que se desenvuelven en su quehacer pedagógico (Alvis-Puentes, Aldana-Bermúdez \& Caicedo-Zambrano,
2019; Castro-Robles, Niño-Vega \& Fernández-Morales, 2020). Por otra parte, la implementación de las TIC como estrategia didáctica, permite que el docente desarrolle escenarios $\mathrm{o}$ implemente herramientas digitales que le permita despertar el interés y motivación a los estudiantes por aprender, al igual que brinda mecanismos para que al estudiante le sea fácil la adquisición de conocimientos, desarrolle habilidades y destrezas entorno a un saber.

Algunos autores como Galeano-Becerra y otros (2018), Garzón-Saladen y RomeroGonzález (2018), Ordóñez-Ortega y otros (2019), han desarrollado e implementado estrategias didácticas mediadas por TIC con el fin de estimular el razonamiento lógico, desarrollar competencias comunicativas y digitales, así como sensibilizar sobre problemáticas alusivas al medio ambiente, y al mejoramiento en la abstracción del conocimiento de áreas disciplinares de los educandos. Los autores manifiestan que la estrategia didáctica mediada por TIC es efectiva, siempre y cuando se identifiquen las dificultades que se les presentan a los educandos, se plantee una ruta de acción pedagógica en donde se tenga en cuenta los contenidos, el ambiente y los recursos educativos de acuerdo con las necesidades de los estudiantes (Niño-Vega, MoranBorbor \& Fernández- Morales, 2019; Angarita-López, 2020). Finalmente, los autores indican la importancia de validar dichas investigaciones con el fin de reevaluar y corregir falencias y optimizar la estrategia desarrollada (Niño-Vega, Martínez-Díaz \& Fernández-Morales, 2016; Salcedo-Ramírez, 2017; Vesga-Bravo \& Escobar-Sánchez, 2018).

\subsection{Competencia comunicativa}

Las competencias comunicativas resultan ser la agrupación de saberes y habilidades las cuales 
posibilitan emitir y captar la información de manera apropiada. Para lograr desarrollar esta competencia es necesario el uso de un lenguaje común, el cual pueda ser empleado como instrumento de comunicación oral o escrita.

Suarez-Ramírez (2019), afirma que para que se garantice una verdadera competencia comunicativa, no solamente se debe desarrollar dicho conjunto de conocimientos y capacidades, sino también se debe desarrollar las competencias: gramatical la cual es requerida para comprender y expresar claramente una idea; sociolingüística, para adaptar las ideas en un contexto determinado; discursiva, para emitir una idea con coherencia y cohesión; y estratégica para compensar los fallos que se pueden presentar en la comunicación.

Becerra, Álvarez, y Rodríguez (2019), establecen que la competencia comunicativa es una capacidad que comprende no sólo la habilidad lingüística, gramatical, de producir frases bien construidas y de saber interpretar y emitir juicios sobre frases producidas por el hablante oyente o por otros, sino que, necesariamente, constará, por un lado, de una serie de habilidades extralingüísticas interrelacionadas, sociales y semióticas, y por el otro, de una habilidad lingüística polifacética y multiforme

Con lo expuesto se puede afirmar que la competencia comunicativa es un factor importante en cuanto a la creación de ambientes que ayuden a los estudiantes a aprender los contenidos del currículum de una forma constructivista y esto permita el desarrollar la habilidad de expresión (Mercer, 2010; Gràcia, Vega, \& Galván-Bovaira, 2015; Gràcia et al.,2017)

\subsection{Competencia digital}

La Comisión Europea (2006, p.15) afirma que la competencia digital es aquella que encamina hacia el uso creativo, seguro y crítico de las TIC, para los diferentes usos de la vida cotidiana. Sin embargo, para poder desarrollar dicha competencia, es necesario el uso apropiado de dispositivos tecnológicos para obtener, evaluar, almacenar, producir, presentar e intercambiar información y comunicarse y participar en redes de colaboración vía internet.

Por otra parte, para poder desarrollar la competencia digital es necesario adquirir conocimientos relacionados con el lenguaje especifico básico alusivo a lo textual, numérico, icónico, visual, gráfico y sonoro (GutiérrezPorlán, Prendes-Espinosa y Martínez- Sánchez, 2018).

Se dice que cuando se logra desarrollar competencias digitales, se logra desarrollar destrezas relacionadas a la búsqueda de la información, el uso y manejo de herramientas tic, la creación y curación de contenidos, la seguridad y protección de datos y la capacidad de resolver problemas reales haciendo uso de recursos tecnológicos (MinTIC, 2016).

\subsection{Competencia narrativa}

Las competencias narrativas son un conjunto de habilidades que permiten relatar historias a partir de recuerdos, representaciones mentales y de experiencia propia. Para poder desarrollar este tipo de competencia, resulta ser necesario involucrar funciones cognitivas, sociales, expresivas y lingüísticas (Figàs-Segura, 2020).

Con el desarrollo de las competencias narrativas, se logra identificar en como la persona percibe y codifica y expresa sus ideas, por tanto, esta competencia permite desarrollar las relaciones interpersonales y favorece su participación en un entorno social (PSISE, 2018).

\subsection{Metodología}

Este estudio se desarrolló bajo un enfoque mixto, debido a que se empleó el enfoque cuantitativo para medir a través de datos numéricos el nivel 
de desempeño obtenido por cada estudiante ante las competencias comunicativas, narrativas y digitales. En cuanto al enfoque cualitativo, se basó en la opinión de los estudiantes frente a la estrategia didáctica desarrollada. El alcance de esta investigación es cuasi experimental, ya que se identifica los hábitos de lectura que tienen los estudiantes, se diseña una estrategia didáctica para mejorarlos y se analiza el nivel de impacto que tuvo esta, sin embargo, no existe aleatorización para la selección de los estudiantes.

La investigación se realizó en la institución educativa Colegio Andrés Rosillo, CAR, ubicado en Bosa de la ciudad de Bogotá. En cuanto a la población objeto de estudio correspondió a 74 estudiantes de los grados cuartos de primaria, de los cuales 39 eran mujeres y 35 hombres, con un rango de edades entre 8 a 10 años. La muestra correspondió a la totalidad de estudiantes de este nivel educativo, debido a que en este nivel, los estudiantes reconocen e interactúan con dispositivos tecnológicos al igual que ya saben leer, comprender y sintetizar información, así como exponer, debatir y argumentar de forma oral o escrita.

Para este estudio se consideró como metodología el desarrolló de tres etapas las cuales son: diseño, desarrollo y validación de la estrategia didáctica basada en el booktrailer.
Etapa 1. Diseño de la estrategia didáctica: se realiza teniendo en cuenta los lineamientos curriculares propuestos por el Ministerio de Educación para la enseñanza de español, al igual que las competencias propuestas en la Guía 30 establecida para la asignatura de tecnología e informática. En esta etapa se establece el ambiente de desarrollo, la duración del curso y las diferentes fases a desarrollar en el aula de clases.

Etapa 2. Desarrollo de la estrategia didáctica: en esta etapa se ejecuta cada una de las fases propuestas en el diseño de la estrategia, teniendo en cuenta que la intervención pedagógica se lleva a cabo en dos asignaturas: español y tecnología e informática.

Etapa 3: Validación de la estrategia didáctica: en esta etapa se aplica la rúbrica diseñada y validada por Del Moral et al. (2016), el cual establece 26 ítems para medir el nivel de desempeño obtenido por los estudiantes en cuanto a las competencias comunicativas, narrativas y digitales, esta medición se hace bajo la escala de valoración: Superior (4.5 5.0), Alto (4.0-4.4), Medio (3.0-3.9) y Bajo (02.9). Igualmente, en esta etapa se realizó una encuesta para identificar el nivel de satisfacción y motivación que tuvieron los estudiantes frente a la estrategia didáctica desarrollada. 


\section{RESULTADOS Y DISCUSIÓN}

\subsection{Diseño y desarrollo de la estrategia didáctica}

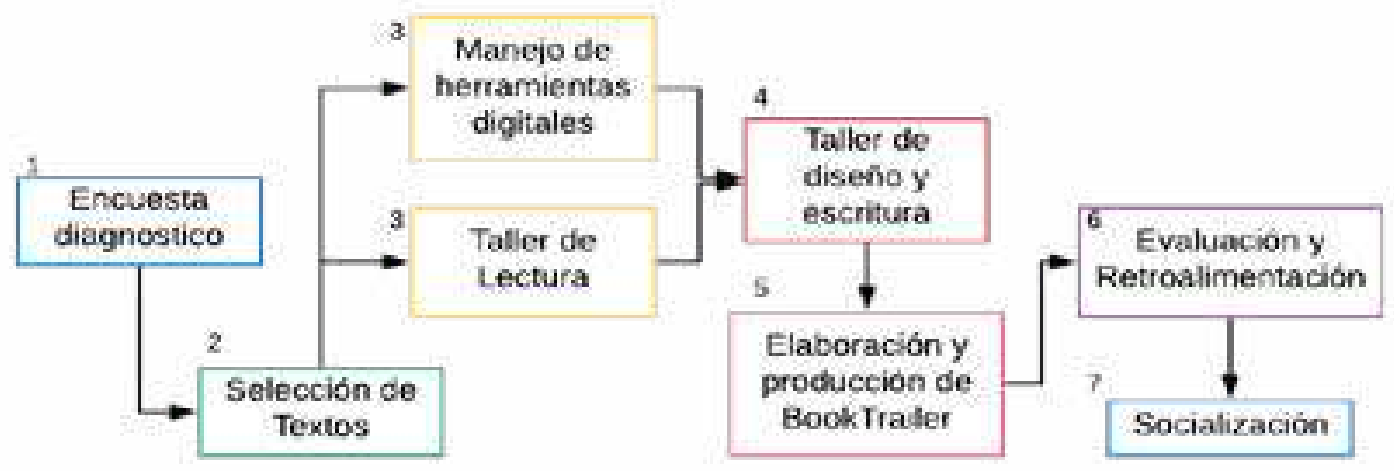

Figura 1. Diseño de la estrategia didáctica

En la figura 1 se establece el diseño de la estrategia didáctica propuesta para el desarrollo de la temática en el aula de clases. En el diagrama se refleja que la actividad 1 , consistió en realizar una encuesta diagnostica para determinar los hábitos de lectura que tienen los estudiantes. En la encuesta se evidenció que: tan solo 13 de los 74 estudiantes dedican al menos una hora diaria de lectura, 67 estudiantes indican conocer y manipular diferentes herramientas tecnológicas digitales, 41 estudiantes manifiestan elaborar textos narrativos para relatar sucesos o exponer ideas, y los 74 estudiantes indicaron contar y saber interactuar con dispositivos tecnológicos tales como computador, celular o tablet, además cuentan con conectividad a internet.

Posteriormente, en la figura 1 se plantea la actividad 2 de selección de libros, para ello se propuso que los estudiantes seleccionaran un libro que no hayan leído con anterioridad y que lo dispongan ya sea de manera digital o impreso, esto con el fin que una vez concluido con la actividad, pueda prestarlo a los demás compañeros que estén interesados en leerlo. Algunos libros seleccionados fueron: Clementina, Dinosaurios al atardecer: Serie la casa del árbol, el misterio del ojo esmeralda,
Cuentos en verso para niños perversos, diario de Greg: vieja escuela, entre otros.

En la actividad 3 se establecen las actividades talleres de lectura y el manejo de herramientas digitales. En cuanto a la actividad de talleres de lectura, esta se realizó en la asignatura de español, en donde cada estudiante dedicó 1 hora de lectura de su libro seleccionado en el aula de clases y de manera extracurricular el estudiante debía terminar de leer el libro en un promedio de 2 meses.

Paralelamente a las lecturas, la actividad de manejo de herramientas digitales se desarrolló en la clase de tecnología e informática, esta actividad consistió en enseñar a los estudiantes a manejar la herramienta StoryboardThat para la realización de escenas y animaciones de sus historietas, los procesadores de texto como Word y Bloc de notas, para la elaboración de guiones y libretos, Paint y Gimp para la elaboración y edición de imágenes, Audacity para la grabación y producción de audios, Avidemux para editar videos con efectos especiales, pistas de audio y textos descriptivos y finalmente Storybird, para la elaboración de ebooks o cuentos animados.

La actividad 4 consistió en la aplicación de un 
taller de diseño y escritura. En esta actividad se les orientó a los estudiantes sobre las técnicas idóneas para la elaboración de videos, teniendo en cuenta el diseño de escenas y el guion para ejecutarlo.

Como paso a seguir, se establece la actividad 5 correspondiente a la elaboración y producción del Booktrailer. Como requisito de esta actividad, los estudiantes debían haber culminado con la lectura del libro y haber aprendido a manejar las herramientas digitales que se les oriento en la actividad 3. En esta actividad, los estudiantes pusieron en practica los conocimientos adquiridos en el curso y elaboraron un video no mayor a 5 minutos en el cual presentaron la sinopsis de lo que consistió el libro, protagonizando escenarios, personajes y trama de la historia.

Enlaactividad6deevaluaciónyretroalimentación, a los estudiantes se les revisó el avance de su booktrailer y se les dieron sugerencias de forma y/o de fondo para mejorar, es decir, se les dio recomendaciones que le permitieron mejorar la calidad del video, imágenes, textos y audios, así como se les revisó la coherencia del mensaje que querían transmitir.

Finalmente, en la actividad 7 se les pidió a los estudiantes que socializaran su booktrailer y para ello se realizó un cinemaraton en donde todos los estudiantes se reunieron y visualizaron el trabajo elaborado por ellos, esto con la intención de despertar el interés y motivación por leer alguno de los demás libros que se presentaron en los videos.

\subsection{Validación de la estrategia didáctica}

En este apartado se presentan los resultados obtenidosa través de los instrumentos empleados para la recolección de datos, ellos permiten evaluar el nivel competencia comunicativa, narrativa y digital obtenido por los estudiantes entorno al desarrollo del Booktrailer para fomentar la lectura en el aula. A continuación, se muestran los resultados obtenidos mediante la rúbrica de evaluación para cada uno de los ítems planteados en cada competencia.

La tabla 1 evidencia el nivel de desempeño obtenido por cada estudiante en cuanto a la competencia comunicativa. En la tabla se valoraron dos aspectos generales para esta competencia los cuales son la competencia comunicativa escrita y oral.

En cuanto al aspecto de la competencia comunicativa escrita se valoraron seis ítems dentro de los cuales, para el desempeño superior el ítem con mayor número de estudiantes fue la utilización correcta de notaciones gráficas, alcanzado por 44 de 74 estudiantes. Esto refleja que los estudiantes reconocen y hacen uso de los signos de puntuación en la redacción de textos. Por otra parte, 27 estudiantes se ubican en el nivel de desempeño alto en el ítem de registro lingüístico acorde con la narración, esto indica que los estudiantes identifican como escribir un cuento, un chiste o una anotación. Igualmente, los resultados permiten evidenciar que a 15 estudiantes se les dificulta la elaboración escrita de los diálogos de los personajes, ya que obtuvieron un nivel de desempeño medio y tan solo 5 estudiantes presentaron problemas con el uso de vocabulario apropiado y por ende su desempeño fue bajo.

Con respecto a la competencia comunicativa oral se valoran seis ítems en donde la plasmación emocional y afectiva del relato y la entonación e inflexión de voz acorde con la temática, fueron los dos ítems alcanzados por la mayoría de los estudiantes, 56 y 50 con un nivel de desempeño superior. Los ítems con mayor número de estudiantes (18) ubicados en el desempeño alto fueron el perfecto ensamblaje de imagen-locución y el ritmo apropiado de interacción de los dialogos entre personajes. Igualmente, los resultados permiten evidenciar que a 16 estudiantes se les dificultó alcanzar el 
ítem de correspondencia entre dialogos y gestos de los personajes por tal razón su desempeño fue medio y para 4 estudiantes el desempeño en este ítem fue bajo.

Tabla 1. Desempeños de los estudiantes en la competencia comunicativa

\begin{tabular}{|c|c|c|c|c|c|}
\hline Competencia & Indicadores & Superior & Alto & Medio & Bajo \\
\hline \multirow{6}{*}{$\begin{array}{l}\text { Comunicativa } \\
\text { escrita }\end{array}$} & $\begin{array}{l}\text { Elaboración escrita de los diálogos } \\
\text { de los personajes. }\end{array}$ & 36 & 20 & 15 & 3 \\
\hline & Uso de vocabulario apropiado & 41 & 17 & 11 & 5 \\
\hline & Corrección gramatical y ortográfica & 43 & 22 & 7 & 2 \\
\hline & $\begin{array}{l}\text { Selección de tiempos verbales } \\
\text { correspondientes a la acción }\end{array}$ & 36 & 26 & 9 & 3 \\
\hline & $\begin{array}{l}\text { Utilización correcta de notaciones } \\
\text { gráficas (signos de puntuación) }\end{array}$ & 44 & 20 & 8 & 2 \\
\hline & $\begin{array}{l}\text { Registro lingüístico acorde con la } \\
\text { narración (cuento, chiste...) }\end{array}$ & 32 & 27 & 9 & 6 \\
\hline \multirow{6}{*}{$\begin{array}{l}\text { comunicativa } \\
\text { oral }\end{array}$} & $\begin{array}{l}\text { Perfecto ensamblaje imagen- } \\
\text { locución (voz en off) }\end{array}$ & 42 & 18 & 10 & 4 \\
\hline & $\begin{array}{l}\text { Correspondencia entre diálogos y } \\
\text { gestos de los personajes }\end{array}$ & 39 & 15 & 16 & 4 \\
\hline & $\begin{array}{l}\text { Ritmo apropiado de interacción de } \\
\text { los diálogos entre personajes }\end{array}$ & 39 & 18 & 16 & 1 \\
\hline & $\begin{array}{l}\text { Dicción y pronunciación adecuada } \\
\text { de los diálogos }\end{array}$ & 51 & 13 & 8 & 2 \\
\hline & $\begin{array}{l}\text { Entonación e inflexión de voz } \\
\text { acorde con la temática }\end{array}$ & 50 & 14 & 7 & 3 \\
\hline & $\begin{array}{l}\text { Plasmación emocional y afectiva del } \\
\text { relato }\end{array}$ & 56 & 12 & 5 & 1 \\
\hline
\end{tabular}

Fuente: elaboración propia.

En la tabla 2 se reportan los resultados de los estudiantes por niveles de desempeño frente a la competencia narrativa. El número total de ítems valorados para esta competencia fueron de 6 en donde la consistencia de los personajes en el booktrailer y la intención comunicativa visible, fueron los dos ítems alcanzados por 59 y 58 estudiantes en nivel superior. Esto indica que los estudiantes logran identificar al personaje y su estado de ánimo dentro de la historia. Por otra parte, 14 estudiantes obtuvieron un nivel de desempeño alto en el ítem de coherencia interna del relato, debido a que lograron identificar las secciones de presentación nudo y desenlace de la trama. Sin embargo, hubo 8 estudiantes a quienes se les dificulto superar este ítem y su nivel de desempeño fue medio. De igual modo es de resaltar que tan solo 4 de los 74 estudiantes tuvieron problemas para la planificación adecuada de escenarios y discurso y por tal razón su desempeño fue bajo, esto falencia se evidencia en el momento en que los estudiantes relatan una historia, pero se desvían del tema central por temas secundarios que no tienen relevancia alguna. 
Tabla 2. Desempeños de los estudiantes en la competencia narrativa

\begin{tabular}{llcccc}
\hline Competencia & \multicolumn{1}{c}{ Indicadores } & \multicolumn{1}{c}{ Superior } & Alto & Medio & Bajo \\
\hline & $\begin{array}{l}\text { Inclusión armónica de los puntos de inflexión } \\
\text { del libro }\end{array}$ & 54 & 13 & 6 & 1 \\
$\begin{array}{l}\text { Coherencia interna del relato (presentación, } \\
\text { nudo, desenlace) }\end{array}$ & 49 & 14 & 8 & 3 \\
$\begin{array}{l}\text { Consistencia de los personajes en el } \\
\text { Narrativa }\end{array}$ & 59 & 13 & 2 & 0 \\
& $\begin{array}{l}\text { Booktrailer } \\
\text { Planificación adecuada de escenarios y }\end{array}$ & 52 & 12 & 6 & 4 \\
$\begin{array}{l}\text { discurso } \\
\text { Resolución creativa de la trama propuesta } \\
\text { Intención comunicativa visible. }\end{array}$ & 54 & 11 & 7 & 2 \\
& 58 & 9 & 6 & 1 \\
\hline
\end{tabular}

Fuente: elaboración propia.

En la tabla 3 se ilustran los niveles de desempeños alcanzados por los estudiantes con respecto a la competencia digital. En esta competencia se evaluaron 8 ítems en el cual, los 74 estudiantes lograron obtener un nivel de desempeño superior en el ítem de movilidad y posturas de los personajes pertinentes a la acción. Esto refleja que los estudiantes lograron dominar las herramientas digitales en cuanto a la edición de los personajes, el cual les posibilito darle gestos, acciones y efectos que hacen que el personaje actúe y se desenvuelva de manera natural. Igualmente, 15 estudiantes obtuvieron un nivel desempeño alto en cuanto al ítem cohesión del sonido y onomatopeyas con la narración. Esto no solo demuestra que los estudiantes realizaron excelentes efectos visuales en cuanto a movilidad, sino que también realizaron sonidos pertinentes a las acciones que se ejecutan.

Por otra parte, el ítem de selección adecuada de planos y angulación de la cámara presento inconvenientes a 6 estudiantes y por tal razón su nivel de desempeño fue medio. Esto se evidencia en el momento en que se presentó el booktrailer, hubo escenas en donde los personajes fueron desenfocados o no estuvo dentro del plano. Por último, 6 estudiantes obtuvieron un nivel de desempeño bajo en el ítem de especificación del proceso creativo, esto se comprobó en la actividad 4 en donde los estudiantes tuvieron problema para plasmar sus ideas sobre el storyboard y en los guiones de la historia. 
Tabla 3. Desempeño de los estudiantes en la competencia digital

\begin{tabular}{|c|c|c|c|c|c|}
\hline Competencia & Indicadores & Superior & Alto & Medio & Bajo \\
\hline \multirow{8}{*}{ Digital } & $\begin{array}{l}\text { Uso adecuado de las aplicaciones } \\
\text { informáticas y sus herramientas }\end{array}$ & 67 & 7 & 0 & 0 \\
\hline & $\begin{array}{l}\text { Estructuración cuidada del proyecto } \\
\text { audiovisual }\end{array}$ & 60 & 7 & 5 & 2 \\
\hline & $\begin{array}{l}\text { Asignación correcta de personajes y } \\
\text { escenarios }\end{array}$ & 62 & 9 & 2 & 1 \\
\hline & $\begin{array}{l}\text { Movilidad y posturas de los personajes } \\
\text { pertinentes a la acción }\end{array}$ & 74 & 0 & 0 & 0 \\
\hline & $\begin{array}{l}\text { Selección adecuada de planos y } \\
\text { angulación de cámara }\end{array}$ & 55 & 13 & 6 & 0 \\
\hline & $\begin{array}{l}\text { Cohesión del sonido y onomatopeyas } \\
\text { con la narración }\end{array}$ & 51 & 15 & 5 & 3 \\
\hline & $\begin{array}{l}\text { Ritmo del relato oportuno para facilitar su } \\
\text { comprensión }\end{array}$ & 56 & 14 & 4 & 0 \\
\hline & $\begin{array}{l}\text { Especificación del proceso creativo } \\
\text { (making off) }\end{array}$ & 49 & 15 & 4 & 6 \\
\hline
\end{tabular}

Fuente: elaboración propia.

En la tabla 4 se evidencia el nivel de desempeño obtenido de los estudiantes en cada una de las competencias propuestas a desarrollar a través de la implementación del booktrailer. Los resultados nos permiten comprobar que las competencias fueron alcanzadas por un gran numero de estudiantes en el nivel superior, entre ella la que más se destaca es la competencia digital con 59 estudiantes. Esto se debe a que los estudiantes utilizan en su diario vivir las herramientas digitales y por tanto demostraron tener un gran dominio en el uso de estas.

Por otra parte, los resultados ilustran que la competencia comunicativa, fue la que presento un mayor numero de estudiantes (3) en el desempeño bajo, frente a las otras dos competencias a evaluar. Esto se debe a que a los estudiantes se les dificultó expresar sus ideas de manera oral en cuanto al relato de sus historias y escrita en los guiones elaborados.

Tabla 4. Desempeño de los estudiantes por competencias

\begin{tabular}{|l|c|c|c|c|}
\hline Competencia & Superior & Alto & Medio & Bajo \\
\hline Comunicativa (escrita y oral) & 42 & 19 & 10 & 3 \\
\hline Narrativa & 54 & 12 & 6 & 2 \\
\hline Digital & 59 & 10 & 3 & 2 \\
\hline
\end{tabular}

Fuente: elaboración propia. 
En cuanto a la validación del curso, se realizó una encuesta a los 74 estudiantes del grado cuarto del CAR el cual tuvo por objetivo conocer las expectativas generadas, la motivación y las actitudes frente al desarrollo de este tipo de actividades para la motivación por la lectura a través de la generación de narrativas digitales para desarrollar competencia comunicativa, digital y literaria mediante Booktrailer. Los resultados de dicha encuesta permitieron denotar que frente a la pregunta ¿te sientes motivado para leer mas libros?, 72 estudiantes contestaron que, si ya que les permite conocer mas historias para narrarlas, así como se entretienen mucho ya que les posibilita imaginar como se desenvuelve el relato y como son los personajes y lugares que se describen en las obras.

En cuanto a la pregunta ¿tuvo algún problema con el manejo de las herramientas digitales?, 69 estudiantes indicaron que no ya que las herramientas eran fáciles de manipular $\mathrm{y}$ muy intuitivas. Por otra parte, 71 estudiantes indicaron que van a seguir explorando más herramientas informáticas las cuales le permitan representar sus ideas, obras y representaciones artísticas en entornos digitales.

Con respecto a la estrategia didáctica del booktrailer en el aula de clases, los estudiantes manifestaron que les fue de mucho agrado debido a que aprendieron a manejar diferentes herramientas digitales para la elaboración de recursos multimedia: adquirieron gusto por la lectura y lograron recrear escenas digitales de lo que cada uno interpreto de la lectura del libro que selecciono. Igualmente, los estudiantes resaltan que gracias al booktrailer, lograron identificar varios libros los cuales llamó su atención. Además, esto demuestra que el realizar un Booktrailer para intrigar a los espectadores sobre el libro, hace que este tenga un valor agregado para la puesta en práctica de sus destrezas, conocimientos y habilidades.

\subsection{Discusión}

Los resultados de este estudio permitieron denotar que el desarrollo de narrativas digitales y booktrailer en el aula es una estrategia pertinente que mejora el desarrollo de competencias comunicativas, narrativas y digitales en los estudiantes, así como fomentar buenas prácticas de lectura y uso de herramientas tecnológicas de los que disponen los entornos virtuales. En este sentido los beneficios de las narrativas digitales a través del desarrollo de booktrailer son bastantes como el incentivar los procesos de lecturas en los estudiantes para obtener un impacto positivo en el desarrollo del futuro profesional, la estimulación del intercambio de información y conocimientos y el aumento de motivación por aprender debido a la implementación de las tecnologías de la información y la comunicación entre otras.

Con respecto a la valoración que se hizo a cada una de las competencias planteadas en este estudio, se puede decir que competencia digital fue la competencia mejor desarrollada por parte de los estudiantes, seguida de la competencia narrativa y la competencia comunicativa oral y escrita. Esto comprueba que al igual que en el trabajo investigativo de Del Moral, Villalustre y Neira (2016), los estudiantes tienden a desarrollar mejor la competencia digital debido a que son nativos digitales y su capacidad de percepción y operabilidad de las herramientas informáticas son excelentes.

\section{CONCLUSIONES}

La investigación aquí rexportada tuvo la finalidad de desarrollar competencias comunicativas, narrativas y digitales a estudiantes de cuarto grado de primaria. Los resultados obtenidos en el estudio permitieron denotar que los estudiantes lograron adquirir estas competencias, luego de haber sido orientados bajo una estrategia didáctica basada en el booktrailer. 
En cuanto a la implementación del booktrailer como estrategia didáctica, el estudio permitió corroborar que esta estrategia logró incentivar a los estudiantes el interés por la lectura, al igual que mejoro la capacidad de análisis, síntesis y argumentación de los estudiantes. Del mismo modo, la implementación de la estrategia didáctica permitió a los estudiantes desarrollar habilidades en cuanto al dominio y uso de herramientas digitales para la elaboración de recursos multimedia a través de softwares de acceso libre.

Se concluye que la implementación del booktrailer como estrategia didáctica, es válida no solamente para fomentar interés y desarrollar competencias lectoescritoras en los estudiantes, sino también para desarrollar competencias digitales a través de la exploración de herramientas digitales. Por tanto, la estrategia didáctica propuesta puede ser empleada en las asignaturas de comprensión lectora y en la asignatura de tecnología e informática.

\section{REFERENCIAS BIBLIOGRÁFICAS}

Alvis-Puentes, J. F., Aldana-Bermúdez, E., \& Caicedo-Zambrano, S. J. (2019). Los ambientes de aprendizaje reales como estrategia pedagógica para el desarrollo de competencias matemáticas en estudiantes de básica secundaria. Revista de Investigación, Desarrollo e Innovación, 10 (1), 135-147.

Angarita-López, R. D., Fernández-Morales, F. H., Niño-Vega, J. E., Duarte, J. E., \& GutiérrezBarrios, G. J. (2020). Accesibilidad de las revistas colombianas del área de humanidades bajo las pautas WCAG 2.1. Revista Espacios, 41 (4), 18. Recuperado de: http://www.revistaespacios.com/ a20v41n04/20410418.html
Avella-lbáñez, C. P., Sandoval-Valero, E. M., \& Montañez-Torres, C. (2017). Selección de herramientas web para la creación de actividades de aprendizaje en Cibermutua. Revista de investigación, Desarrollo e Innovación, 8(1), 107-120.

Barrera-Mesa, C. E., Fernández-Morales, F. H., \& Duarte, J. E. (2017). Diseño de un ambiente de aprendizaje mediado por TIC para la enseñanza de operadores mecánicos orientado al grado séptimo de la educación básica, en el Colegio Boyacá de Duitama. Revista Colombiana de Tecnologías de Avanzada, 2 (30), 1119.

Barrera-Mesa, M., Fernández-Morales, F. H., \& Duarte, J. E. (2017). Aprendizaje basado en proyectos colaborativos mediados por TIC para el desarrollo de competencias en estadística. Saber, Ciencia y Libertad, 12 (2), 220-232. Recuperado de: http:// www.sabercienciaylibertad.org/ojs/index. php/scyl/article/view/247

Becerra, S. P., Álvarez, W. O., \& Rodríguez, A. A. (2019). Competencias comunicativas para la vida a través del uso de la multimedia. Revista Espacios, 40(20), 17.

Bernate, J. A., García-Celis, M. F., FonsecaFranco, I. P., \& Ramírez-Ramírez, N. E. (2020). Prácticas de enseñanza y evaluación en una facultad de educación colombiana. Revista de Investigación, Desarrollo e Innovación, 10 (2).

Castro-Robles, A. D., Niño-Vega, J. A., \& Fernández-Morales, F. H. (2020). El acoso escolar como oportunidad para mejorar la convivencia en las aulas. Educación y Humanismo, 22 (38), 1-13. doi: 10.17081/ eduhum.22.38.3675 
Cruz-Rojas, G. A., Molina-Blandón, M. A., \& Valdiri-Vinasco, V. (2019). Vigilancia tecnológica para la innovación educativa en el uso de bases de datos y plataformas de gestión de aprendizaje en la universidad del Valle, Colombia. Revista de Investigación, Desarrollo e Innovación, 9 (2), 303-317.

Figàs-Segura, M. (2020). Narrativas audiovisuales colectivas como vehículo de resignificación identitaria: implementando el vídeo participativo con jóvenes guatemaltecos/as. Revista Internacional de Comunicación y Desarrollo (RICD), 3(11), 80-91. https://doi.org/10.15304/ ricd.3.11.6381

Fonseca-Barrera, C. C., Niño-Vega, J. A., \& Fernández-Morales, F. H. (2020). Desarrollo de competencias digitales en programación de aplicaciones móviles en estudiantes de noveno grado a través de tres estrategias pedagógicas. Revista Boletín Redipe, 9(4), 179-191. https://doi. org/10.36260/rbr.v9i4.958

Galeano-Becerra, C. J., Bellón-Monsalve, D., Zabala-Vargas, S. A., Romero-Riaño, E., \& Duro-Novoa, V. (2018). Identificación de los pilares que direccionan a una institución universitaria hacia un SmartCampus. Revista de Investigación, Desarrollo e Innovación, 9 (1), 127-145. doi: https://doi.org/10.19053/20278306. v9.n1.2018.8511

Garzón-Saladen, Á., \& Romero-González, Z. (2018). Los modelos pedagógicos y su relación con las concepciones del derecho: puntos de encuentro con la educación en derecho. Revista de Investigación, Desarrollo e Innovación, 8(2), 311-320.
Gràcia, M., Jarque, M. J., Astals, M., Rouaz, K., \& Tovar, C. (2017). La competencia comunicativa y lingüística oral en la formación inicial de maestros: un estudio piloto. CIAIQ 2017, 1.

Gràcia, M., Vega, F., \& Galván-Bovaira, M. J. (2015). Developing and testing EVALOE: a tool for assessing spoken language teaching and learning in the classroom. Child Language Teaching and Therapy, 31 (3), 287-304

Gutiérrez - Porlán, I., Prendes-Espinosa, M. P., \& Martinez-Sanchez, F. (2018). Competencia digital una necesidad del profesorado universitario en siglo XXI. RED Revista de Educación a Distancia, 56(7), 1-22. DOI:http://dx.doi. org/10.6018/red/56/7

Hernández-Gil, C., \& Jaramillo-Gaitán, F. A. (2020). Laboratorio de innovación social: hibridación creativa entre las necesidades sociales y las experiencias significativas de los estudiantes de administración de empresas. Revista de Investigación, Desarrollo e Innovación, 10 (2).

Jiménez-Espinosa, A. (2019). La dinámica de la clase de matemáticas mediada por la comunicación. Revista de Investigación, Desarrollo e Innovación, 10 (1), 121-134.

Jiménez-Espinosa, A., \& Sánchez-Bareño, D. M. (2019). La práctica pedagógica desde las situaciones a-didácticas en matemáticas. Revista de Investigación, Desarrollo e Innovación, 9 (2), 333-346.

López-Gaitán, M. A., Moran-Borbor, R. A., \& Niño-Vega, J. A. (2018). Prácticas experimentales como estrategia didáctica para la comprensión de conceptos de física mecánica en estudiantes de educación superior. Infometric@ - Serie Ingeniería, Básicas y Agrícolas, 1 (1), 
1-14. Recuperado de: http://infometrica. org/index.php/syh/article/view/12

Martínez-López, L. G., \& Gualdrón-Pinto, E. (2018). Fortalecimiento del pensamiento variacional a través de una intervención mediada con TIC en estudiantes de grado noveno. Revista de Investigación, Desarrollo e Innovación, 9 (1), 91-102.

Mercer, N. (2010). The analysis of the classroom talk: Methods and methodologies. British Journal of Educational Psychology, 1, 1-14. Doi: https://doi. org/10.1348/000709909X479853

Ministerio de Tecnologías de la Información y Comunicaciones. (2016), Currículos exploratorios, MINTIC. Recuperado de: http://aprende.colombiaaprende.edu.co/ es/curriculostic

Naranjo-Gaviria, A. A., \& Herreño-Téllez, E. (2020). Caracterización de las actividades interdisciplinarias en una facultad de artes Colombiana. Revista de Investigación, Desarrollo e Innovación, 10 (2).

Niño-Vega, J. A., \& Fernández- Morales, F. H. (2019). Una mirada a la enseñanza de conceptos científicos y tecnológicos a través del material didáctico utilizado. Revista Espacios, 40 (15). Recuperado de: http://www.revistaespacios.com/ a19v40n15/19401504.html

Niño-Vega, J. A., Fernández-Morales, F. H., \& Duarte, J. E. (2019). Diseño de un recurso educativo digital para fomentar el uso racional de la energía eléctrica en comunidades rurales. Saber, Ciencia y Libertad, 14 (2), 256-272. doi: https://doi.org/10.18041/2382-3240/ saber.2019v14n2.5889
Niño-Vega, J. A., Martínez-Díaz, L. Y., \& Fernández-Morales, F. H. (2016). Mano robótica como alternativa para la enseñanza de conceptos de programación en Arduino. Revista Colombiana de Tecnologías de Avanzada, 2 (28), 132-139. doi: https://doi.org/10.24054/16927257. v28.n28.2016.2476

Niño-Vega, J. A., Martínez-Díaz, L. Y., Fernández-Morales, F. H., Duarte, J. E., Reyes-Caballero, F., \& Gutiérrez-Barrios, G. J. (2017). Entorno de aprendizaje para la enseñanza de programación en Arduino mediado por una mano robótica didáctica. Revista Espacios, 38 (60), 23. Recuperado de: http://www.revistaespacios.com/ a17v38n60/17386023.html

Niño-Vega, J. A., Moran-Borbor, R.A., \& Fernández- Morales, F. H. (2019). Educación inclusiva: Un nuevo reto para la labor docente en el siglo XXI. Infometric@ - Serie Sociales y Humanas, 2 (1), 74-94. Recuperado de: http://infometrica.org/ index.php/ssh/article/view/78

Ordóñez-Ortega, O., Gualdrón-Pinto, E., \& Amaya-Franky, G. (2019). Pensamiento variacional mediado con baldosas algebraicas y manipuladores virtuales. Revista de Investigación, Desarrollo e Innovación, 9 (2), 347-362.

PSISE. (2018). Las competencias narrativas. Servicio de Psicología Clínica del Desarrollo. Unidad de Observación y Diagnóstico Funcional. Recuperado de: https://psisemadrid.org/competenciasnarrativas/ 
Ruiz-Macías, E., \& Duarte, J. (2018). Diseño de un material didáctico computarizado para la enseñanza de Oscilaciones y Ondas, a partir del estilo de aprendizaje de los estudiantes. Revista de Investigación, Desarrollo e Innovación, 8(2), 295-309.

Salcedo-Ramírez, R. Y., Fernández-Morales, F. H., \& Duarte, J. E. (2017). Unidad didáctica para la enseñanza de probabilidad mediada por un OVA, orientada a un colegio rural del municipio de Paipa. Revista Colombiana de Tecnologías de Avanzada, 2 (30), 1-10.

Salcedo-Salcedo, S. P., \& Fernández, F. (2018). Mejoramiento de la escritura en inglés como lengua extranjera en niños con síndrome de Down mediada por las TIC. Revista Boletín Redipe, 6(11), 76-85. Recuperado de: https://revista.redipe.org/ index.php/1/article/view/402

Suarez-Ramírez, M. (2019). Libro de texto, práctica educativa y competencia comunicativa/Textbook, educational practice and communicative competence. Polyphōnía: Revista de Educación Inclusiva/Polyphōnía: Inclusive Education Journal, 3(1).

Vargas-Vargas, N. A., Niño-Vega, J. A., \& Fernández-Morales, F. H. (2020). Aprendizaje basado en proyectos mediados por tic para superar dificultades en el aprendizaje de operaciones básicas matemáticas. Revista Boletín Redipe, 9(3), 167-180. https://doi.org/10.36260/ rbr.v9i3.943

Vesga-Bravo, G. J., \& Escobar-Sánchez, R. E. (2018). Trabajo en solución de problemas matemáticos y su efecto sobre las creencias de estudiantes de básica secundaria. Revista de Investigación, Desarrollo e Innovación, 9 (1), 103-114. 